\title{
Un enfoque sobre la medición y nuestra disciplina
}

\author{
Capozzo, Marcela*; Gómez Mengelberg, Elizabeth**
}

\section{Resumen}

El propósito de este trabajo es promover la reflexión, en una disciplina de carácter esencialmente social, como la Terapia Ocupacional, sobre el rol que juega lo mensurable y el peligro que atañe considerar lo cuantitativo y la formalización como las únicas formas válidas de producción del conocimiento.

Esta reflexión se inscribe en el debate epistemológico actual de las ciencias sociales, que focaliza en los aspectos cualitativos y cuantitativos, la medición, la formalización y sus alcances.

El método utilizado para aproximarnos y examinar esta temática es el de la búsqueda y revisión bibliográfica, y el análisis de los conceptos centrales de la temática sobre la cual nos planteamos la presente indagación.

Palabras clave: Conocimiento- cualitativo-cuantitativo-investigación-objetividad-medición

\section{Summary}

The intention of this work is to promote the reflection, in a discipline of essentially social character, like the Occupational Therapy, on the roll that the mensurable thing plays and the danger that concerns to consider quantitative and the formalization as the only valid forms of production of the knowledge.

This reflection registers in the present epistemologic debate of social sciences, that focus in the qualitative and quantitative aspects, the measurement, the formalization and its reaches.

The used method to approximate to us and to examine this thematic one is the one of the search and bibliographical revision, and the analysis of the central concepts of the thematic one on which we considered the present investigation.

Key words: Knowledge- qualitative-quantitative- investigation- objectivity-measurement

\footnotetext{
* Licenciada en Terapia Ocupacional- Jefa de Sección del Servicio de Terapia Ocupacional -Hospital Interdisciplinario Psicoasistencial Dr. José T. Borda -Buenos Aires - Especialista en Metodología de la Investigación

**Licenciada en Terapia Ocupacional- Jefa de Sección del Servicio de Terapia Ocupacional -Hospital

Interdisciplinario Psicoasistencial Dr. José T. Borda -Buenos Aires -Especialista en Metodología de la InvestigaciónDocente de la Universidd Nacional de General San Martín.

Contacto > > Capozzo, Marcela: E-mail: marcelacapozzo@yahoo.com.ar

**Gómez Mengelberg, Elizabeth: E-mail: egmengelberg@fibertel.com.ar

Ramón Carrillo 375, Ciudad de Buenos Aires, Argentina

Fono: 011 4305-5083
} 


\section{- Introducción "}

El llevar a la reflexión el rol que juega lo mensurable y el peligro que atañe a considerar lo cuantitativo y la formalización como la única forma válida de producir conocimiento, en una disciplina de carácter esencialmente social como la Terapia Ocupacional, es el propósito de este trabajo.

Dentro de las diferentes clasificaciones posibles de la ciencia, hallamos la que permite referirse a estas como: Ciencias formales (lógica, matemática) Ciencias naturales (física, química, biología) y sociales o humanas (economía, sociología, psicología, antropología, historia, comunicación, etc.

Recurriendo a los diferentes niveles de la semiótica o teoría de los signos, podría destacarse que las ciencias formales son de carácter primordialmente sintáctica, dado que se privilegia la relación de los signos sin importar lo que éstos representan, mientras que las ciencias naturales son semánticas, ya que existe una relación entre los signos y aquello que representan. Por otra parte, las ciencias sociales o humanas son pragmáticas, entendiendo lo pragmático en este caso en el sentido del papel más relevante del individuo o usuario en relación con la ciencia de la que se trate, es por ello que está presente, la relación de los signos, aquello que representan y los usuarios.

Desde otra perspectiva, Eliseo Verón las diferencia según la menor o mayor participación de los sujetos investigadores, consecuentemente designa ciencias de baja pertenencia a las naturales y de alta pertinencia a las sociales y humanas ${ }^{(\underline{1})}$.

Otro eje que es frecuentemente enfatizado con relación a clasificar a las diferentes ciencias, es aquel que destaca el carácter fáctico o empírico de las mismas. En este sentido en las ciencias naturales la confrontación de sus enunciados con la experiencia para confirmarlos o refutarlos adquiere un carácter central y distintivo, no sucede lo mismo con las ciencias formales de primacía sintáctica. Las ciencias sociales pueden considerarse fácticas, por cuanto se ocupan de hechos, de sus propios hechos. ${ }^{(1)}$

Por otra parte, el área de conocimiento y la práctica de la Terapia Ocupacional, es el interés por los problemas del hombre en su vida de actividades. En otras palabras, considera a las actividades humanas como un producto y un medio de construcción propio del hombre, que busca entender las relaciones que este hombre en actividad, establece en su condición de vida y de salud ${ }^{(2)}$.

Este interés se va desarrollando bajo los paradigmas que se constituyen en el ámbito de las comunidades científicas. En 1962 se publico un libro llamado La estructura de las revoluciones científicas del filosofo epistemológico estadounidense Tomas Kuhn que sacudió la modorra de la epistemología anglosajona tuvo la osadía de introducir la noción de historia en una reflexión sobre la ciencia, es decir propone analizar el fenómeno de la ciencia desde su devenir histórico, al decir de Khun un paradigma se constituye a partir de las realizaciones científicas universalmente reconocidas, las que durante un tiempo proporcionan modelos de conocimiento a una comunidad científica determinada, las investigaciones no se pueden llevar a cabo sin marcos referenciales propios de cada época científico - histórica ${ }^{(\underline{3})}$. Michel Foucault no es epistemólogo es un filosofo de la cultura, por lo tanto no analiza la ciencia en si misma sino como acontecimiento histórico-cultural para la visión de Foucault la actividad científica conforma un dispositivo de saber-poder avalado por la racionalidad de sus discursos y 
de sus prácticas, y no hay verdad inamovible que perdure en el tiempo, son más importantes- aunque no desestima la decisión de la comunidad científica- las condiciones de posibilidad para que unos discursos accedan al status de verdaderos, en detrimento de otros, y pone énfasis en los factores de poder de cualquier cuño; económico, educativo, científico y otros, donde se asientan las condiciones de posibilidad. ${ }^{(5,6)}$. Se establece entonces un territorio teórico que se llama episteme moderno.

Si bien la episteme de Focault (condiciones de posibilidad) es científico-social y la noción de paradigma de Khun es sólo científico, ambos conceptos de Focault y de Khun son epocales y ambos conforman una especie de marco teórico o imaginario social para la producción de discursos considerados verdaderos en un determinado momento histórico. $(\underline{7})$

Khun especifica que las ciencias sociales no gozaron nunca de un paradigma aceptado universalmente por la comunidad científica, y Foucault declara que estas disciplinas tienen un perfil epistemológico más débil que las naturales, y es más fácil descubrir las relaciones de poder que subyacen en la conformación de los conceptos y las prácticas científicas. La posibilidad de formalizar matemáticamente sus enunciados y de corroborarlos con la experiencia, es lo que históricamente se denomina un perfil epistemológico fuerte, como las ciencias naturales. ${ }^{(\underline{7})}$

Khun nos advirtió que existen presiones mucho más poderosas que la inocente validez formal de una teoría científica dijo "ganan las teorías que tienen fuerza, no las más verdaderas"(3) y Foucault se sumergió en el mundo de las prácticas sociales, los intereses, el deseo y la lucha de poder, intereses económicos, corporativos, personales y cognoscitivos que sostienen esa aparentemente inofensiva estructura formal de las teorías científicas. ${ }^{(6)}$

El debate epistemológico en el que actualmente se encuentra el área de conocimiento de Terapia Ocupacional sus límites y alcances abreva en la práctica que se presenta constituida por modelos y técnicas de intervención oriundas de diferentes concepciones asumidas por las ciencias en las que se fue basando la práctica (biología, psicología, antropología y otros) y valoraciones del hombre y de sus actividades, formando una amalgama teórico-práctica que se ha ido modificando a lo largo de la historia.

Este debate no es ajeno al que se ha venido desarrollando en las diferentes ciencias sociales y humanas, e implica la premisa de que cualquier discusión epistemológica se basa en una sentencia filosófica y antropológica.

Desde su nacimiento, en las Ciencias sociales y humanas, la discusión se ha centrado sobre tres ejes: el objeto de estudio, el método y el estatus epistemológico. En torno a ellos se han desarrollado, desde el siglo XIX a la actualidad, dos concepciones: la naturalista y la posnaturalista. Basadas en ellas, se establecieron diferentes posturas que conviven en la práctica de la investigación hoy ${ }^{(\underline{8})}$.

Dentro de la concepción naturalista empirista se homologa el mundo social al físico, entendiendo a ambos como estructuras invariantes donde es posible encontrar regularidades empíricas. Esto deriva en la afirmación de que existe una sola manera de hacer ciencia, la que corresponde al método de las ciencias naturales, ubicadas en el segundo nivel en el orden de la semiótica o sea el semántico 
La investigación científica está orientada a la búsqueda de explicaciones, que adquieren las forma de leyes generales que dan cuenta de fenómenos particulares, y el conocimiento científico es o pretende ser fundamentalmente objetivo.

Frente al desarrollo de la vertiente naturalista se halla la reacción comprensivista que surge planteando la especificidad de lo social como irreductible; en consecuencia esgrime el dualismo metodológico que se ubica como un hiato entre las ciencias sociales y las naturales.

Desde esta perspectiva el conocer en ciencias sociales no será subsumir fenómenos particulares mediante leyes, sino comprender, siendo este proceso alcanzado desde la propia subjetividad del intérprete.

A partir de la década del sesenta entra en crisis la concepción estándar de la filosofía de la ciencia en sus diferentes vertientes, lo que trae aparejado un replanteo en la perspectiva desde la cual se debate sobre las ciencias en general y consecuentemente, en las sociales y humanas.

El escenario del Siglo XXI se plantea entonces, posnaturalista, siendo una de sus principales características el lenguaje, comprendido ya no como un medio de comunicación, un instrumento para intermediar la relación del hombre con las cosas, sino como materia prima del mundo social, como un horizonte último de la inteligibilidad de los procesos históricos y sociales. Se reeduca la visión del científico que se desplaza del fenómeno visible del hecho social de los positivistas a la estructura del lenguaje y el carácter esencialmente lingüístico de las acciones humanas.

Se plantea también una concepción ampliada de la razón y la ciencia, dado que se reconoce la existencia de otras formas de racionalidad además de la técnico instrumental desarrollada y afianzada desde el proyecto filosófico de la modernidad.

Se destaca el supuesto hermenéutico que implica la ruptura con el supuesto empirista en que se basa la visión naturalista. El plural escenario postnaturlista y postempirista pone el acento en la inevitable carga teórica de los enunciados científicos, esto significa que ya no es posible hablar de observación pura o de datos en sentido estricto.

En consecuencia el mundo social sobre el que ejerce su acción el científico, es un mundo ya interpretado por los actores sociales. ${ }^{(\underline{8})}$

El pluralismo se refleja asimismo en la dimensión interpretativa e intersubjetiva de la verdad. Frente a la concepción tradicional de la verdad como adecuación de un enunciado a la realidad, comienzan a aparecer intentos por construir una idea de verdad alternativa. Es decir la verdad entendida desde otras perspectivas como la hermenéutico-interpretativa, la dimensión histórica, las teorías consensuales (al modo de los paradigmas) o la teoría de las acciones comunicativas.

Otra característica de este escenario postnaturalista, es la de pertenencia del intérprete a una tradición, es decir que siempre se comprende desde la pertenencia a un mundo en el cual estamos y que posee siempre un sentido. Es por ello que todo saber que se precie de científico deberá ejercer cierto control sobre posibles proyecciones, pero es importante es destacar la presencia de una preestructura del comprender que se anticipa a toda objetividad, a toda distanciación, ya que será desde ella que se produzca el conocimiento y la comprensión de la realidad. 
La impronta positivista en la producción de las ciencias sociales y humanas que incluye la producción de conocimiento en Terapia Ocupacional y su reflejo en las investigaciones sobre de la misma, nos lleva a preguntarnos sobre el fuerte impulso y el nivel de adhesión alcanzado por lo mensurable y la formalización por parte de los colegas. Esto se observa en el creciente interés por obtener instrumentos de medición que permitan conocer y categorizar acerca de las actividades y la ocupación humana, transformándolas a través de la aplicación de métodos formales (matemáticos, estadísticos, modelización) en entes que trascienden lo contextual e histórico. Además de reducir la complejidad de la actividad y ocupación, una de las características de la riqueza y vastedad del campo disciplinar de la Terapia Ocupacional.

Dentro de las ciencias naturales la medición es uno de sus baluartes, análogamente las ciencias sociales y humanas que se inscriben dentro de esta tradición defienden la creencia de entidades que se exteriorizan y que pueden ser mensurables. Son los cultores de posiciones epistemológicas formalistas y empiristas que consideran que sólo lo cuantitativo es serio y desprecian las categorías cualitativas sin darse cuenta que sin ellas cualquier medición sería imposible ${ }^{(\underline{9})}$.

Cuando se señala que las ciencias sociales son fácticas no estamos sosteniendo que para serlo deban ajustarse a un modelo físico de realidad sino que han de configurar su propia realidad $\stackrel{(\underline{10})}{ }$.

Puede sostenerse que la facticidad y la posibilidad de confrontación están estrechamente vinculadas y esto es relevante también para las ciencias sociales, que indudablemente plantearán la cuestión en su propio ámbito, con autonomía, aunque sin descuidar las conexiones que resulten pertinentes $(\underline{10})$.

La descripción de la realidad social por parte de las ciencias sociales debe extenderse ante el hecho de que ese conocimiento de la realidad procede no sólo de las ciencias sociales sino del pensamiento normativo y de la producción cultural ${ }^{(\underline{9})}$.

El uso más frecuente de la investigación social se refiere a la absorción de ciertas ideas y determinados conceptos creados por ella por parte de los grupos que adoptan decisiones políticas.

Puede señalarse entonces, una interrelación entre las transformaciones sociales y las que van produciéndose en las ciencias sociales $\stackrel{(\underline{10})}{ }$.

La investigación social es una forma de conocimiento que se caracteriza por la construcción de evidencia empírica elaborada a partir de la teoría, aplicando reglas de procedimiento explícitos. En su contenido la investigación es temporal -histórica, acotada y acumulativa, está sujeta a inexactitudes $\mathrm{y}$, por lo tanto, es parcial 0 totalmente refutable.

Este carácter de temporal-histórica está dado porque los temas que trata y cómo los trata están profundamente afectados por las circunstancias históricas, mundiales y locales; por los intereses económicos y sociales y las ideas predominantes en las instituciones patrocinantes, en institutos de investigación, en los ámbitos académicos y entre sus miembros ${ }^{(10)}$.

Tomas Khun sostiene que la medición ha funcionado exitosamente respaldando las técnicas cuantitativas por basarse en la ilusión de que las mediciones se producen en 
circunstancias a-históricas alejadas de cualquier sesgo psicológico, cultural o político, esto contribuye a la idea de la objetividad y de universalidad.

Aún hay epistemólogos y científicos que siguen exigiendo que las ciencias sociales reduzcan sus accionar al de las ciencias naturales, abrazan una intención reduccionista al pretender que todas las disciplinas con pretensión de cientificidad deberían seguir un modelo físico-matemático ${ }^{(\underline{9})}$.

La medición y la contrastación contienen ambigüedades, desajustes y preconceptos, es decir soportan anomalías. Al no existir la teoría perfecta la medición tampoco lo es. Lo empírico existe concretamente, lo intelectual, elabora explicaciones, interpretaciones acerca de la realidad. Se crea una medición y luego se decreta que ese procedimiento responde al orden natural de las cosas, disponiendo que si algo es formalizable; es sólido, verdadero y creíble, esta es una interpretación posible pero su autoproclamación como verdad la convierte en dogmática.

Observamos en nuestro campo disciplinar la Terapia Ocupacional, una insistencia imperiosa- enarbolada preferentemente por los profesionales mas noveles; de realizar mediciones, cuantificar, formalizar matemáticamente, quizás a instancias de necesitar demostrar al mundo científico la efectividad de los tratamiento realizados en TO o la eficacia de la disciplina misma, o abonar la creencia de que algo mensurable y formalizado matemáticamente de por si cuenta con un aval de cientificidad difícil de poner en duda, otra respuesta posible es la existencia de un espíritu sectario que tiene su cuota de poder manejando lenguajes herméticos hasta a veces con pretensión pseudocientíficas, reservándose entonces el manejo de los objetos formalizados y la exclusión del terreno científico de lo no cuantitativo, escamoteándole a las ciencias sociales y humanas la solidez que se merecen.

Es quizás un intento de construir con relación al objeto de estudio de terapia ocupacional, una abstracción, plausible de ser universalizada, al estilo del modelo positivista y siguiendo con la concepción tradicional de la ciencia moderna, sin considerar el concepto de episteme, del que nos habla Foucault, presente en cualquier producción de conocimiento.

Es probable que la dificultad traducida en imposibilidad de tolerar la angustia de transitoriedad del escenario particular y singular de la vida de cada sujeto según su historia comunitaria y personal los lleve a aferrarse en este mar embravecido de la balsa de las mediciones, de los standares, y arriesgarse a extrapolar aquí lo realizado y validado en otras realidades histórico socio-económicas.

El grado de desventaja de un sujeto depende no sólo del ambiente humano que lo rodea sino también del no humano que lo rodea y señala la posibilidad de intervenir sobre ese ambiente.

Quizás estudios epidemiológicos propios, criterios y categorizaciones latinoamericanas nos ayuden a acercarnos a un perfil más acorde a lo que somos.

Aún así, la medición por sí sola no nos dice nada, los números no hablan por sí mismos carecen de carga semántica necesitan de referencias explícitas, ya que sin interpretaciones, sin alusiones a lo que se quiere modelizar, no expresan nada significativo. 
Las ciencias naturales y sociales deberían acudir a la medición como parámetro necesario pero no suficiente, y no deberían perder de vista y estar alerta que siempre se entrelazan con algún poder, explícito o subyacente, para reducir el riesgo de un posible sesgamiento.

Lo cualitativo es lo que da respaldo a lo cuantitativo. Las mediciones en sí mismas no poseen intencionalidad, por lo cual no se las puede valorar positiva o negativamente, sino más bien deben responder a fundamentos no sólo teóricos, sino a la historicidad del sujeto evaluado y además ser objeto de revisiones constantes para no caer en el dogmatismo ${ }^{(\underline{9})}$.

\section{Consideraciones finales "}

Asumir la tensión de la relación que se produce entre los polos cualitativo y cuantitativo, no implica una resolución en uno u otro sentido como posturas antagónicas de la producción de conocimiento científico.

A quien se le realice una medición, una evaluación, un test, un cuestionario, una encuesta es de hecho y de derecho un sujeto social con una historia particular, dentro de un contexto en una sociedad determinada y en una cultura que incidirán de manera manifiesta o latente en las respuestas dadas y el que lleva adelante estas acciones es un profesional que interviene en el campo del otro, con su propia historicidad y subjetividad. Tener en cuenta esto es respaldar y ampliar la base de sustentación de la perspectiva mensurable y cuantitativa, tener en cuenta las condiciones de posibilidad de las acciones permite un acercamiento menos sesgado.

Finalmente nos parece importante señalar que la asunción de cualquier posición dogmática se convierte en un obstáculo para el progreso de la ciencia.

\section{- Referencias "}

(1) Eliseo Verón. 1977. "Ideología y comunicación de masas: la sistematización de la violencia política" Ed. Nueva Visión.

(2) Da Rocha Medeiros. M.H- 2003- "Terapia ocupacional, um enfoque epistemológico e social" Editora HUCITEC- Edufscar- Sao Paulo.

(3) Khun T.S. 1978 "Segundo pensamiento sobre paradigmas" Madrid. Ed. Tecnos.

(4) Khun, T.S. 1990 "La estructura de las revoluciones científicas" Buenos Aires.

FCE 
(5) Foucault, M. 1975 "Las palabras y las cosas" México. Ed Siglo Veintiuno, 1978

(6) Foucault, M. 2005 "La verdad y las formas jurídicas" Barcelona, Ed Gedisa.

(7) Díaz Esther - 2000 "La Posciencia- El conocimiento científico en las postrimerías de la modernidad" Editorial Biblos. Buenos Aires.

(8) PARDO R. H- El desafío de las ciencias sociales. Del Naturalismo a la hermeneútica. En: Perspectivas Metodológicas- Revista anual del Depto. de Humanidades y artes de la Universidad Nacional de Lanús .2003; No III, 5155

(9) Schuster. F.G- 1997. "El método en las ciencias sociales" Editores de América Latina- Argentina. I. 1-13

(10) DIAZ E.-Las ciencias sociales, lo mensurable y la sombra del poder. En: Cuadernos de trabajo del Centro de Investigaciones en teorías y prácticas científicas del Depto de Humanidades y Artes de la Universidad Nacional de Lanús. 2003; No II; 4-17. 